\title{
Treatment of Unexplained Infertility by Acupuncture in Natural and Control Ovarian Hyperstimulation Cycles: A Prospective Analysis
}

\author{
Liuhong Cai ${ }^{*}$, Rihan Hai², Bin Zhang³, Yanfei Wen'1, Minhui Zeng1, Manbo Jiang ${ }^{3}$ \\ ${ }^{1}$ Center for Reproductive Medicine, The Third Affiliated Hospital, Sun Yat-sen University, Guangzhou, China \\ ${ }^{2}$ Department of Acupuncture, The Third Affiliated Hospital, Sun Yat-sen University, Guangzhou, China \\ ${ }^{3}$ Department of Infertility and Sexual Medicine, The Third Affiliated Hospital, Sun Yat-sen University, \\ Guangzhou, China \\ Email: ${ }^{*}$ cailh@mail.sysu.edu.cn
}

Received 29 September 2014; revised 30 October 2014; accepted 10 November 2014

Copyright (C) 2014 by authors and Scientific Research Publishing Inc.

This work is licensed under the Creative Commons Attribution International License (CC BY). http://creativecommons.org/licenses/by/4.0/

(c) (i) Open Access

\begin{abstract}
Acupuncture is an important method of treatment in Chinese medicine. The objective of this study was to evaluate the efficacy of acupuncture as an adjuvant treatment for unexplained infertility. Here we conducted a prospective study, with data consisting of acupuncture group (38 cases) and control group (42 cases). Infertility evaluation workup consisted of semen analysis, ovulation assessment, hysterosalpingogram (HSG) and blood analysis. The patients in acupuncture group received 3 acupuncture sessions, and each at seven acupuncture points (EX-CA1, CV4, CV6, SP10, ST36, SP6, and KI3). The session started 12 days before menstruation and continued for 10 days. The patients in control group did not receive acupuncture. All patients tried 1 - 3 natural cycles 3 months after HSG test, if not pregnant, underwent 1 - 3 cycles of control ovarian hyperstimulation $(\mathrm{COH})$ and timed intercourse. Pregnancy was evaluated by measurement of blood $\beta$ human chorionic gonadotrophin ( $\beta$-hCG) and subsequent trans-vaginal ultrasound. No significant difference of clinical pregnancy rate was found between the acupuncture group and the control group, however, numbers of $\mathrm{COH}$ cycles were significantly less and more pregnancies occurred in natural cycle in the acupuncture group. We concluded that acupuncture can be used as an adjuvant treatment for unexplained infertility. Although acupuncture did not increase the cumulative pregnancy rate, it decreased the number of $\mathrm{COH}$ cycles and more patients got pregnant in natural cycles after receiving acupuncture.
\end{abstract}

\footnotetext{
*Corresponding author.
}

How to cite this paper: Cai, L.H., Hai, R.H., Zhang, B., Wen, Y.F., Zeng, M.H. and Jiang, M.B. (2014) Treatment of Unexplained Infertility by Acupuncture in Natural and Control Ovarian Hyperstimulation Cycles: A Prospective Analysis. Advances in Reproductive Sciences, 2, 88-92. http://dx.doi.org/10.4236/arsci.2014.24011 


\section{Keywords}

\section{Acupuncture, Unexplained infertility, Control Ovarian Hyperstimulation, Pregnancy}

\section{Introduction}

Infertility affects $10 \%$ - $15 \%$ of the reproductive age group. According to The Practice Committee of the American Society for Reproductive Medicine (ASRM), after the standard infertility evaluation workup of semen analysis, assessment of ovulation, hysterosalpingogram (HSG), and, if indicated, tests for ovarian reserve and laparoscopy, approximately $15 \%$ to $30 \%$ couples were assigned a diagnosis of unexplained infertility due to no abnormality revealed [1] [2]. For unexplained infertility, intrauterine insemination (IUI) or in vitro fertilization (IVF) can be a good choice, but both lay a heavy economic burden on patients.

Acupuncture is a traditional Chinese treatment, widely practiced and now also accepted in the western world for the treatment of obstetric and gynecologic or infertile issues [3]-[6].

We have been practicing acupuncture for unexplained infertility treatment for many years. In this study we prospectively analyzed the data to evaluate the effect of acupuncture in treatment of unexplained infertility.

\section{Methods}

\subsection{Study Design and Subjects}

This is a prospective study implemented at The Third Affiliated Hospital, Sun Yat-Sen University, between March 2012 and Aug 2013.

The study group eligibility criteria included: infertility duration $\geq 3$ years; age of 22 - 35 years; presence of regular menstrual cycles and ovulation; absence of tubal obstruction on HSG; sperm concentration > 15 million/ $\mathrm{ml}$ and total sperm number $>39$ million/ml according to WHO criteria [1]. All women underwent a traditional Chinese medicine (TCM) diagnosis. Exclusion criteria were the presence of endocrinologic disease (PCOS, etc.); use of nonsteroidal anti-inflammatory drugs or corticosteroids; clinical findings or HSG results suggesting pelvic inflammatory disease; the presence of undiagnosed uterine bleeding; refusal to undergo $\mathrm{COH}$. The patients willing to receive acupuncture were assigned to acupuncture group, otherwise to control group. The study was conducted in accordance with the Declaration of Helsinki, and was approved by the Reproductive Ethics Committee of the Third Affiliated Hospital of Sun Yat-sen University.

\subsection{Workup for Evaluation of Infertility}

Patients were asked to come with their husbands. First, husbands received physical examination and were asked to do semen analysis. Then, wives were asked to monitor ovulation by ultrasound for at least 1 cycle and test hormone on day 2 to day 5 of menstruation cycle. At the same time, antibodies, such as anti-HCG antibodies, anti-sperm antibodies, anti-ovarian antibodies, anti-sperm antibodies, anti-cardiolipin antibodies, anti-zona pellucida antibodies, etc, were tested. Thyroid function, including TSH, FT3, FT4, were tested. Vaginal discharge examination and ultrasound examination of pelvic organs were conducted.

HSG: Patients were scheduled between the 7th and 12th day of their menstrual cycle (3 - 7 days after menstruation). Contraindications included pregnancy, vaginal inflammatory disease and severe allergy against iodine contrast agents. Half an hour before HSG, a spasmolytic agent, atropine, was injected. Antibiotic prophylaxis was prescribed after HSG. The cervical os was cannulated using a catheter with $3 \mathrm{ml}$ balloon. When the catheter was in place, water-soluble iodinated contrast medium was applied under X-ray. An injection of $10 \mathrm{ml}$ contrast medium outlined the cornual, isthmic and ampullary portions of the tubes, and showed contrast medium spillage into the abdominal cavity. If one or both tubes showed no contrast spillage into the abdominal cavity, the possibility of tubal spasm was excluded by increasing injection pressure and contrast medium administration.

Nine radiographs were taken after contrast medium administration in each patient, including images of the early and complete filling phases of the uterine cavity, outlining of the fallopian tubes and the contrast spillage into the abdominal cavity. Delayed image was taken 30 minutes after removal of the instrument from the cervix.

Following the examination, the radiologist evaluated the images independently. 


\subsection{Acupuncture Treatment}

Each patient from acupuncture group underwent a total of 3 acupuncture sessions, each session performed 12 days before menstruation, for 10 days continuously. In all sessions, the treatment was performed by the same acupuncturist, with more than 20 years of experience in acupuncture. The needles were manually inserted bilaterally and stimulated to obtain de qi and kept for 20 minutes. The following points for acupuncture were selected:

EX-CA1: Zigong-On the lower abdomen, 4 cun below the centre of the umbilicus and 3 cun lateral to Zhongji (RN3); CV4: Guanyuan—On the midline, 3 cun inferior to the umbilicus; CV6: Qihai-On the midline, 1.5 cun inferior to the umbilicus; SP10: Xuehai-With the knee in flexion, 2 cun superior to the superior border of the patella, on the bulge of the medial portion of the quadriceps femoris muscle; ST36: Zusanli-3 cun below ST35 one finger width lateral from the anterior crest of the tibia, in the tibialis anterior muscle; SP6: Sanyinjiao3 cun directly above the tip of the medial malleolus on the posterior border of the tibia; KI3: Taixi-In depression midway between the tip of the medial malleolus and the attachment of the Achilles tendon, level with the tip of the medial malleolus.

\subsection{Natural Cycle}

After HSG, both groups were instructed to try up to 3 natural cycles or until pregnancy occurred. They were asked to monitor ovulation and were administered 10,000 IU of hCG (Lizhu Phamacy, China) to trigger ovulation when leading follicle reached a diameter of $18 \mathrm{~mm}$.

\subsection{Controlled Ovarian Hyperstimulation ( $\mathrm{COH})$}

Both groups were instructed to try up to $3 \mathrm{COH}$ cycle. Urinary hMG and hCG were used for ovarian stimulation. Ovulation induction was started between day 2 and day 5 of menstruation cycle in patients who had no residual cysts larger than $20 \mathrm{~mm}$ by basal transvaginal ultrasound monitor. All patients initially received $75 \mathrm{IU} /$ day hMG for 5 days. Follicle growth was measured ultrasonographically every 1 - 5 days. Initial dose was increased or decreased between 37.5 - 75 IU/day according to follicle size and number. Blood estriol, LH and P levels were monitored and recorded on hCG injection day. When 1 - 3 follicles reached a diameter of $18 \mathrm{~mm}, 10,000 \mathrm{IU}$ of hCG was administered to trigger ovulation. Cycle was cancelled when more than 3 mature follicles were observed. Patients conducted timed coitus 36 - 48 hours after the hCG injection. Dydrogesterone Tablets (Solvay Pharmaceuticals) were administered to support the luteal phase for 12 days. Patients with positive hCG received progesterone support until twelve weeks of gestation. If the hCG result was negative, another $\mathrm{COH}$ cycle was initiated.

\subsection{Pregnancy Evaluation}

Patients whose urine test results were positive had a repeat blood test to ascertain a healthy increase in beta hCG levels. An intrauterine gestational sac and presence of fetal cardiac activity confirmed the viable clinical pregnancy. Patients who experienced pregnancy loss after the sac was visualized were considered as clinical miscarriages. Chemical pregnancy was confirmed by the detection of serum $\beta$-hCG on the 14th day after ovulation.

\subsection{Statistical Analysis}

Data were analyzed by SPSS version 13.0 for Windows (SPSS Inc., Chicago, Illinois, USA). Continuous variables (age, duration of infertility, total motile sperm count, initial and total dose of ovulation induction agent, follicle count and size and endometrial thickness) were presented as mean \pm SD. Categorical variables were presented as frequency and percentage. Student's t test was used to compare normally distributed continuous variables and Mann-Whitney $U$ test for variables without normal distribution. Categorical variables were compared using the chi-square test. A two-tailed $\mathrm{p}$ value of $<0.05$ was considered statistically significant.

\section{Results}

Totally 92 case were recruited, from which 12 patients were lost or refused to follow the protocol. From the remaining 80 patients, 42 were in control group and 38 in acupuncture group. The comparison of the two groups is 
summarized in Table 1.

\section{Discussion}

The principal treatments for unexplained infertility include timed intercourse and lifestyle changes, $\mathrm{COH}$, intrauterine insemination (IUI) or in vitro fertilization (IVF) [7]. The procedures of IUI and IVF are more expensive and take longer time.

Acupuncture has always been applied to reproductive treatment in China. Western medicine may exert influence on neuroendocrine system, immunological functions and even signal pathway in consideration when discussing the efficacy of acupuncture and these still continue to be studied. The Chinese medicine evaluates the effect of acupuncture from an overall perspective. In Chinese medicine, reproductive function relates not only to reproductive organs, but also to the kidney, the liver, and the heart .That is why we usually select many acupuncture points besides Zigong when performing treatment. Acupuncture is rooted in traditional Chinese medicine (TCM) and emphasises individualised treatment according to TCM patterns [8], here we modify our acupoints basing on the most frequently used ones, such as sanyinjiao (SP6), Guanyuan (CV4), Zhongji (CV3) and Zigong (Ex-CA1) being.

A meta-analysis of 27 studies involving 2939 cycles revealed that the pregnancy rate per cycle was $8 \%$ with gonadotropin treatment alone and 18\% with gonadotropin treatment combined with IUI [9]. In this study, a cumulative pregnancy rate of $24 \%$ for control group and $29 \%$ for acupuncture group was achieved, and no significant differences were found between the 2 groups. Hysterosalpingography may have contributed partly to the high pregnancy rate. The primary role of HSG is to evaluate the morphology and the patency of the fallopian tubes [10], and has a mechanical washing effect on the uterine cavity and tubes, increasing the chance of spontaneous pregnancy, especially with oil-based contrast. Therefore, following a normal HSG, in our center, we recommended up to 3 cycles as expected duration of spontaneous pregnancy. This was valuable, because 1 couple from control group and 3 from acupuncture group achieved spontaneous pregnancy.

Although no significant differences were found in pregnancy rate achieved by 2 study groups, it was easier for acupuncture group to achieve spontaneous pregnancy in natural cycle. Regarding COH cycles, significantly fewer cycles were needed in acupuncture group. This suggested that though acupuncture did not increase the cumulative pregnancy rate, it did decrease the expected time, both in $\mathrm{COH}$ and natural cycles.

The reason that we need not wait too many cycles also base on studies results. Unexplained infertility also means the possibility that some abnormality has not been detected. For those women with unexplained infertility with at least three failed cycles of $\mathrm{COH}$ and IUI, laparohysteroscopy revealed that pelvic abnormalities were found in 36.7\% women and hysteroscopy showed 6.7\% intrauterine lesions [11]. This kind of patients has relatively low chance of a successful pregnancy, and assisted reproductive technologies should be considered.

Limitation of this study is that the small number of samples, and we need more detailed data about the mechanism of acupuncture.

\section{Conclusion}

The results of this study indicate that acupuncture can be used as an adjuvant treatment for unexplained infertile-

Table 1. Comparison of the two groups.

\begin{tabular}{cccc}
\hline & Control group $(\mathrm{n}=42)$ & Acupuncture group $(\mathrm{n}=38)$ & $\mathrm{p}$ value \\
\hline Age (years) & $28.2 \pm 4.7$ & $32.8 \pm 5.3$ & 0.40 \\
Duration of infertility (years) & $4.9 \pm 1.1$ & $5.1 \pm 1.8$ & 0.45 \\
Primary infertility (cases) & 22 & 13 & 0.102 \\
hMG doses (ampoules) & $12.0 \pm 3.2$ & $12.5 \pm 4.5$ & 0.87 \\
Mature eggs & $1.56 \pm 0.88$ & $1.67 \pm 0.91$ & 0.684 \\
COH cycles & $2.12 \pm 0.56$ & $1.48 \pm 0.37$ & 0.01 \\
Total clinical pregnancy (cases) & 10 & 11 & 0.272 \\
Spontaneous pregnancy (cases) & 1 & 3 & 0.341 \\
Biochemical (cases) & 1 & 1 & 0.728 \\
Miscarriage prior to 12 weeks (cases) & 2 & 0 & 0.495 \\
\hline
\end{tabular}


ity. Although acupuncture did not increase the cumulative pregnancy rate, it decreased the time to achieve pregnancy in $\mathrm{COH}$ cycles.

\section{Funding}

This work was supported by Guangdong Province Science and technology project (2013B021800091).

\section{References}

[1] The Practice Committee of the American Society for Reproductive Medicine (2006) Effectiveness and Treatment for Unexplained Infertility. Fertility and Sterility, 86, S111-S114. http://www.ncbi.nlm.nih.gov/pubmed/17055802 http://dx.doi.org/10.1016/j.fertnstert.2006.07.1475

[2] The Practice Committee of the American Society for Reproductive Medicine (2006) Optimal Evaluation of the Infertile Female. Fertility and Sterility, 86, S264-S267. http://www.ncbi.nlm.nih.gov/pubmed/17055838 http://dx.doi.org/10.1016/j.fertnstert.2006.08.041

[3] Villahermosa, D.I., Santos, L.G., Nogueira, M.B., Vilarino, F.L. and Barbosa, C.P. (2013) Influence of Acupuncture on the Outcomes of in Vitro Fertilisation When Embryo Implantation Has Failed: A Prospective Randomised Controlled Clinical Trial. Acupuncture in Medicine, 31, 157-161. http://aim.bmj.com/content/31/2/157.long http://dx.doi.org/10.1136/acupmed-2012-010269

[4] Smith, C.A. and Betts, D. (2014) The Practice of Acupuncture and Moxibustion to Promote Cephalic Version for Women with a Breech Presentation: Implications for Clinical Practice and Research. Complementary Therapies in Medicine, 22, 75-80. http://www.ncbi.nlm.nih.gov/pubmed/24559820 http://dx.doi.org/10.1016/j.ctim.2013.12.005

[5] Smith, C.A., de Lacey, S., Chapman, M., Ratcliffe, J., Norman, R.J., Johnson, N., Sacks, G., Lyttleton, J. and Boothroyd, C. (2012) Acupuncture to Improve Live Birth Rates for Women Undergoing in Vitro Fertilization: A Protocol for a Randomized Controlled Trial. Trials, 13, 60. http://www.ncbi.nlm.nih.gov/pubmed/22607192 http://dx.doi.org/10.1186/1745-6215-13-60

[6] Isoyama, D., Cordts, E.B., de Souza van Niewegen, A.M., de Almeida Pereira de Carvalho, W., Matsumura, S.T. and Barbosa, C.P. (2012) Effect of Acupuncture on Symptoms of Anxiety in Women Undergoing in Vitro Fertilisation: A Prospective Randomised Controlled Study. Acupuncture in Medicine, 30, 85-88.

http://www.ncbi.nlm.nih.gov/pubmed/22499825 http://dx.doi.org/10.1136/acupmed-2011-010064

[7] Quaas, A. and Dokras, A. (2008) Diagnosis and Treatment of Unexplained Infertility. Reviews in Obstetrics \& Gynecology, 1, 69-76. http://www.ncbi.nlm.nih.gov/pubmed/18769664

[8] Birkeflet, O., Laake, P. and Vøllestad, N. (2012) Traditional Chinese Medicine Patterns and Recommended Acupuncture points in Infertile and Fertile Women. Acupuncture in Medicine, 30, 12-16. http://www.ncbi.nlm.nih.gov/pubmed/22378581 http://dx.doi.org/10.1136/acupmed-2011-010089

[9] Guzick, D.S., Sullivan, M.W., Adamson, G.D., Cedars, M.I., Falk, R.J., Peterson, E.P. and Steinkampf, M.P. (1998) Efficacy of Treatment for Unexplained Infertility. Fertility and Sterility, 70, 207-213. http://www.ncbi.nlm.nih.gov/pubmed/9696208 http://dx.doi.org/10.1016/S0015-0282(98)00177-0

[10] Schankath, A.C., Fasching, N., Urech-Ruh, C., Hohl, M.K. and Kubik-Huch, R.A. (2012) Hysterosalpingography in the Workup of Female Infertility: Indications, Technique and Diagnostic Findings. Insights into Imaging, 3, 475-483. http://www.ncbi.nlm.nih.gov/pubmed/22802083

[11] Firmal, P., Yadav, R. and Agrawal, S. (2014) A Prospective Study to Evaluate the Role of Aparohysteroscopy in Unexplained Infertility. Journal of Obstetrics and Gynaecology. (Epub ahead of print)

http://www.ncbi.nlm.nih.gov/pubmed/25325846 
Scientific Research Publishing (SCIRP) is one of the largest Open Access journal publishers. It is currently publishing more than 200 open access, online, peer-reviewed journals covering a wide range of academic disciplines. SCIRP serves the worldwide academic communities and contributes to the progress and application of science with its publication.

Other selected journals from SCIRP are listed as below. Submit your manuscript to us via either submit@scirp.org or Online Submission Portal.
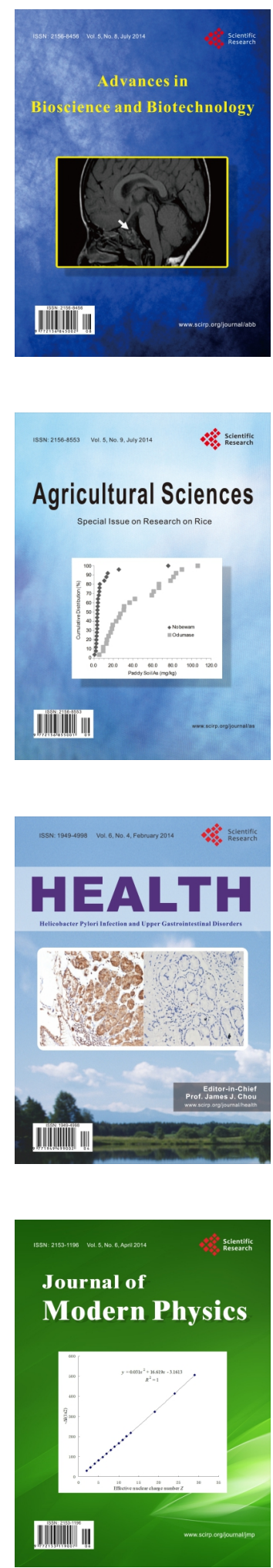
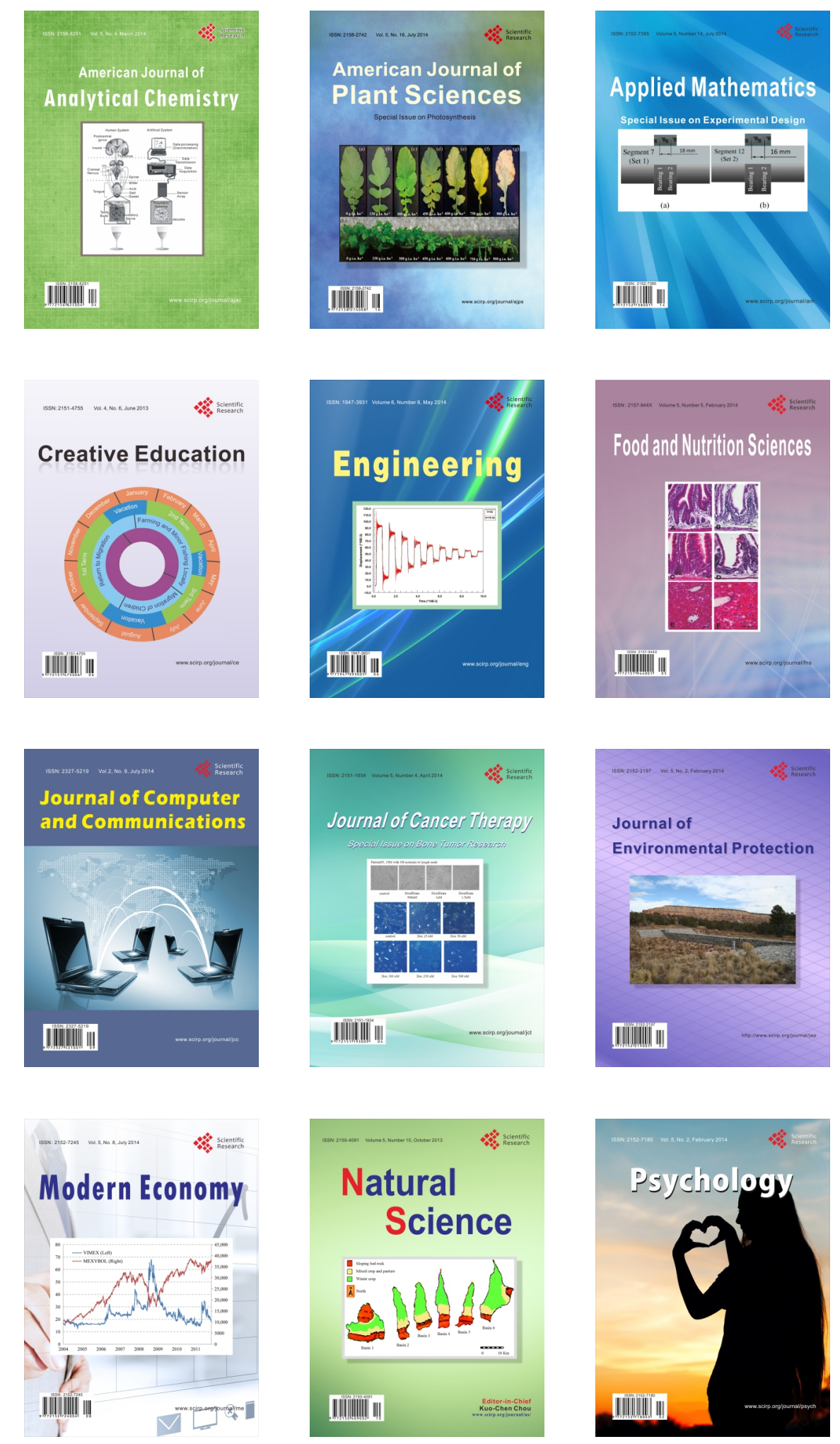